\title{
Comparison of three different methods to measure arterial stiffness and their relationship with age, BMI and central blood pressure
}

\author{
Z. Maniou, P. J. Chowienczyk ${ }^{2}$, W. L. Hall ${ }^{1}$ and T. A. B. Sanders ${ }^{1}$ \\ ${ }^{1}$ Nutritional Sciences Research Division, King's College London, 150 Stamford Street, London SE1 9NN, UK \\ and ${ }^{2}$ Cardiovascular Division, King's College London, St Thomas' Hospital, London SE1 7EH, UK
}

\begin{abstract}
Arterial stiffness has emerged as a strong predictor of risk of cardiovascular events independent of blood pressure ${ }^{(1)}$. The most widely used and validated method to measure large-artery stiffness is the carotid to femoral pulse wave velocity (PWV; PWV (SphygmoCor; AtCor Medical Pty Ltd,West Ryde, NSW, Australia). This method requires finding a pulse in the femoral artery in the groin and has some disadvantages for routine use, as it requires skilled personnel with steady hands to get a reliable reading. Two other techniques have been used to measure arterial stiffness. The simplest of these techniques is to estimate a stiffness index (SI) from the digital wave form (Pulse Trace; MicroMedical Ltd, Basingstoke, Hants., UK) and a more recent device (Vicorder; Skidmore Medical Ltd, Bristol, UK; Vicorder PWV) has claimed to yield results equivalent to those using tonometry; both techniques are quick and do not require skilled operators. Arterial stiffness increases with age ${ }^{(2)}$ but the effect of adiposity or BMI on this measurement is uncertain. The relationship between these variables is reported for 183 subjects (sixty-two male, 121 female; age 44-69 (mean 55) years) recruited into the MARINA study (ISRCTN6666461) for whom the three types of arterial stiffness measurement were carried out at baseline. Subjects attended a clinical research facility in the fasting state and body composition was determined using a BodPod (Life Measurement Inc., Concord CA, USA). Measurements of PWV $\mathrm{c}_{\mathrm{c}-\mathrm{f}}$, Vicorder PWV and SI were carried out on the subjects in the supine position after 15 min rest. Data for PWV and SI were log transformed and Vicorder PWV data required a reciprocal transformation. Spearman's correlations between the Vicorder PWV and SI and the reference $\mathrm{PWV}_{\mathrm{c}-\mathrm{f}}$ were $\rho 0.68$ and $\rho 0.301$ respectively (both $P<0.001$ ). However, the correlation between SI and $\mathrm{PWV}_{\mathrm{c}-\mathrm{f}}$ was stronger for the men than for the women ( $\rho 0.465$ and $\rho 0.198$ respectively). A linear regression model was fitted for the transformed variables for each gender with age, gender, central systolic BP and percentage body fat for the three methods. There was no relationship between body fatness and measures of arterial stiffness determined as PWV $\mathrm{V}_{\mathrm{c}-\mathrm{f}}$ or Vicorder PWV and this relationship was determined mainly by age and central systolic BP. In contrast there was a weak relationship between increased BMI and PWV. The results are shown in the Table.
\end{abstract}

\begin{tabular}{|c|c|c|c|c|c|c|c|}
\hline \multirow[b]{2}{*}{ BMI $\left(\mathrm{kg} / \mathrm{m}^{2}\right) \ldots$} & \multicolumn{2}{|c|}{$20-25$} & \multicolumn{2}{|c|}{$25-30$} & \multicolumn{2}{|c|}{$30-35$} & \\
\hline & Mean & $95 \% \mathrm{CI}$ & Mean & $95 \% \mathrm{CI}$ & Mean & $95 \% \mathrm{CI}$ & \\
\hline $\mathrm{PWV}_{\mathrm{c}-\mathrm{f}}(\mathrm{m} / \mathrm{s})$ & 9.1 & $8.8,9.4$ & 9.4 & $9.1,9.7$ & 10.1 & $9.5,10.6$ & $P=0.011$ \\
\hline Vicorder PWV $(\mathrm{m} / \mathrm{s})$ & 12.5 & $12.0,13.0$ & 12.3 & $11.7,12.9$ & 13.7 & $12.7,14.7$ & $P=0.045$ \\
\hline
\end{tabular}

These findings indicate that the Vicorder PWV gives more reproducible results for measurement of arterial stiffness compared with the SI. One possible reason for the discrepancy between the strength of the relationship between males and females for the SI may be associated with the difficulty in getting a good pulse wave form in women because of cold hands. The mean within-subject SD for the three measures was $0.42 \mathrm{~m} / \mathrm{s}$ with the Vicorder compared with $0.53 \mathrm{~m} / \mathrm{s}$ for the SphygmoCor. The results show no relationship between arterial stiffness and body fatness but were able to discern an effect of BMI on arterial stiffness.

1. Anderson SG, Sanders TA \& Cruickshank JK (2009) Hypertension 53, 839-845.

2. Millasseau SC, Kelly RP, Ritter JM et al. (2002) Clin Sci (Lond) 103, 371-377. 